\title{
Designing Visfatin inhibitors to limit its Insulin Mimcy and Type II Diabetes.
}

Neha Pandya ${ }_{L}{ }^{1}$ Earl Benjamin, ${ }^{1}$ Ellis Benjamin*1

Department of Chemistry, The Richard Stockton College of New Jersey, Galloway NJ 08205-

9441.

Keywords: Visfatin, Type II Diabetes, Inflammation

Visfatin, otherwise known as Nicotinamide phosphoribosyltransferase (NAmPRTase or Nampt), is an adipocytokine that promotes B cell maturation and inhibits neutrophil apoptosis as well as promoting the condensation of nicotinamide. Visfatin plays an important role in promoting insulin resistance by binding to insulin receptor (IR) at a site distinct from insulin exerting a variety of insulin-mimetic effects, thereby playing a role in the development of obesityassociated insulin resistance and Type II diabetes. This research sought to understand binding interaction of pharmaceuticals to Visfatin. 11 crystal structures of the Visfatin were docked using IGEMDock to FDA, Alkaloids, Lactams, Lactones, Flavinoids, Sulfanilamide, Cyclic Imides, and NSAIDs drugs to determine structural correlation for the most effective binders. Structural similarities were determined with IGEMDock and VROCS and partition coefficient was determined using DRAGON program. This data found a cluster of potential inhibitors to Visfatin which are possible targets for Type II diabetes treatments. This research will be used in the engineering of improved Visfatin inhibitors.

\section{Introduction}

This project was designed around structural understanding and pharmaceutical engineering of the Visfatin inhibitors. Visfatin being a fat produced insulin mimic may play a part in the initiation of Type 2 diabetes. Additionally, its interaction as an adipocytokine may be a factor in inflammatory damage in obese individuals. The understanding and limitation of Visfatin may help limit both Type 2 diabetes and inflammatory damage caused by Visfatin.

\section{Specific and Overall Goal}

The overall goal of this research is to investigate the interaction of multiple drug candidates to find the best drug candidates for targeted inhibition of Visfatin. This research will first determine the binding and chemical properties of the Visfatin active site molecules as a control group. Secondly, a group of select drug candidates whose properties are more effective at binding to the active site versus the control molecules will be chosen. Drug classification analysis will indicate preferences to improved active site binding. Finally, quantitative structure and activity relationship (QSAR) analysis will be done on both the control and experimental molecules to identify similar trends and values. Once found, similarities between these drugs will allow for a better understanding of the inhibition of Visfatin. 


\section{Experimental Methodology}

1. The selection of 11 isoforms of Visfatin.

2. The screening and analysis of multiple drug candidates using IGemDock.

3. Similarity calculations were done to determine if molecular functionalities showed any preference to increased binding.

4. Selected candidates from IGemDock based on binding energy were tested using Dragon to determine structural similarities.

\section{Methods and Materials}

1172 structures were chosen which included, 715 FDA approved, 197 Alkaloids, 73 Imides, 40 Lactams, 36 Lactones, 50 NSAIDs, 25 Sulfanilamide and 37 Flavonoids pharmaceuticals were selected as ligands and computationally bound to 11 isoforms of the Visfatin protein using IGEMDock. IGEMDock used two independent docking with the average of both binding to factor in binding selectivity. An ANOVA was done to determine if any discrepancies in binding were seen between proteins. Additionally, grouping of the molecular functionalities were determine to find differences. The best 10 drug candidates on binding energies were selected and structural data such as molecular weight and partition coefficient was collected using Dragon.

\section{Visfatin Crystal Structures from Protein Databank}

\begin{tabular}{c|c|l}
$\#$ & PDB & \multicolumn{1}{|c}{ Title } \\
\hline 1 & 2 E5C & Crystal structure of Human NMPRTase complexed with 5'-phosphoribosyl-1'-pyrophosphate \\
2 & 2 E5D & Crystal structure of Human NMPRTase complexed with nicotinamide \\
3 & $2 \mathrm{G} 96$ & Crystal Structure of Visfatin In Complex with Niconamide Mononucleotide \\
4 & $2 \mathrm{G} 97$ & Crystal Structure of Visfatin In Complex with the Specific Inhibitor FK-866 \\
5 & $2 \mathrm{GVG}$ & Crystal Structure of human NMPRTase and its complex with NMN \\
6 & $2 \mathrm{GVL}$ & Crystal Structure of Murine NMPRTase \\
7 & $2 \mathrm{H} 3 \mathrm{~B}$ & Crystal Structure of Mouse Visfatin \\
8 & $3 \mathrm{DGR}$ & Crystal structure of human NAMPT complexed with ADP analogue \\
9 & $3 \mathrm{DHD}$ & Crystal structure of human NAMPT complexed with nicotinamide mononucleotide and pyrophosphate \\
10 & $3 \mathrm{DKJ}$ & Crystal structure of human NAMPT complexed with benzamide and phosphoribosyl pyrophosphate \\
11 & 3DKL & Crystal structure of human NAMPT complexed with benzamide and phosphoribosyl pyrophosphate
\end{tabular}


Crystal Structure of 2E5C (UCSF Chimera)

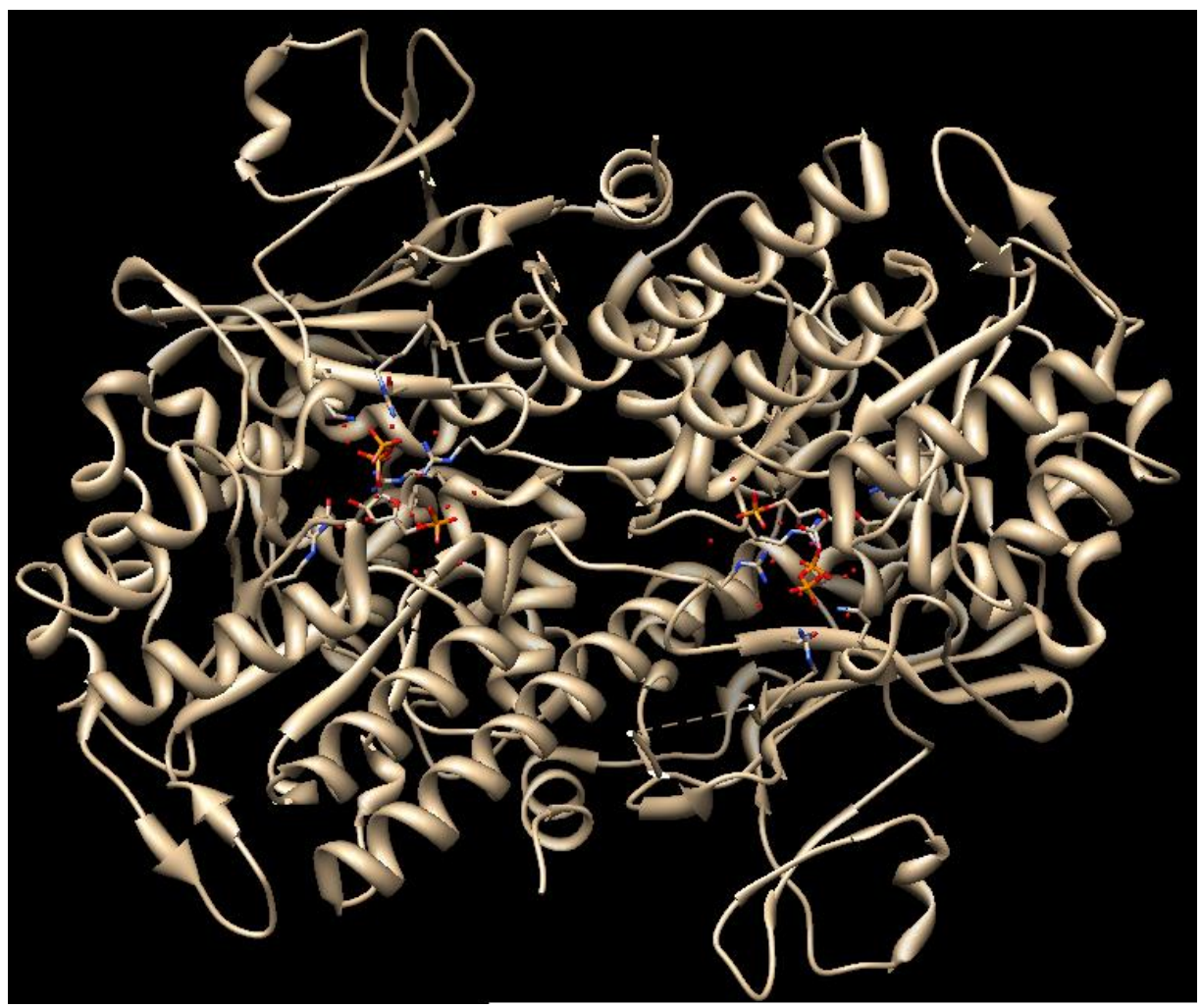

Active Site of 2E5C (UCSF Chimera)

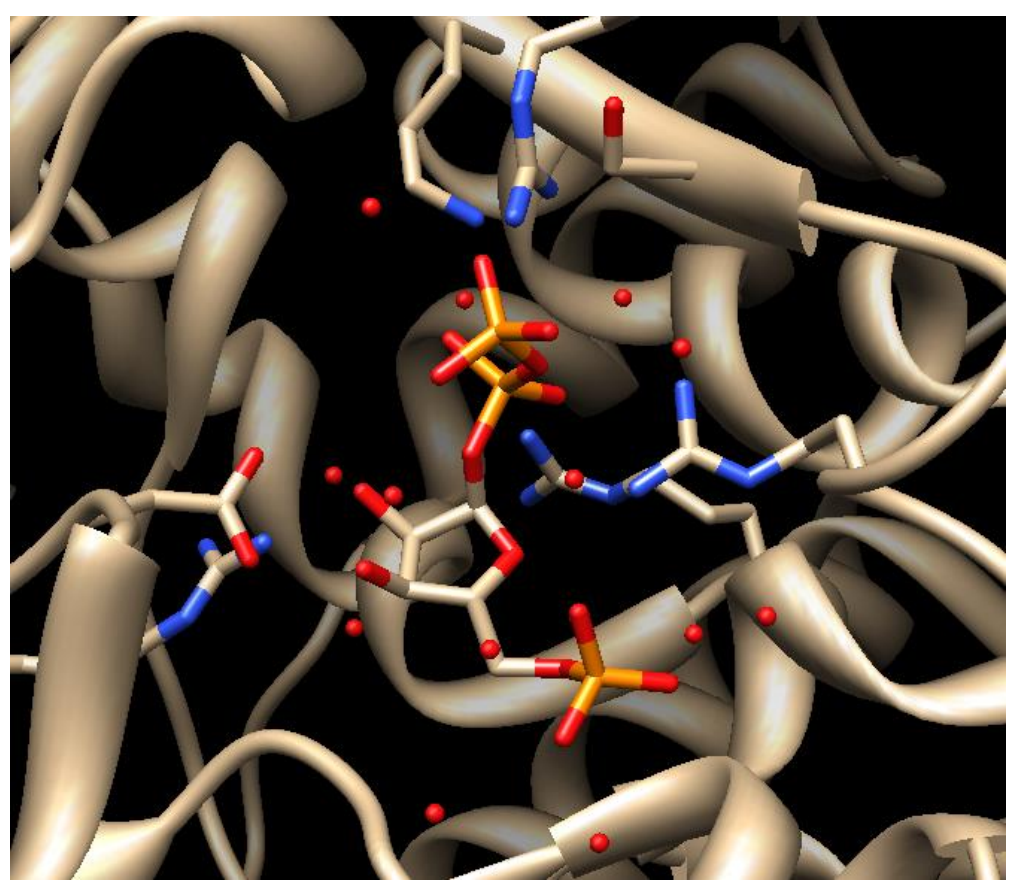


Summary of 1172 Drug Candidates vs Proteins (IGEMDock Data).

\begin{tabular}{ccccc}
\multicolumn{5}{c}{ SUMMARY } \\
\hline Groups & Count & Sum & Average & Variance \\
\hline 2E5C & 2344 & -187874 & -80.1508 & 6019.472 \\
2E5D & 2344 & -172874 & -73.7518 & 5691.071 \\
2G96 & 2344 & -184175 & -78.5729 & 5881.788 \\
2G97 & 2344 & -176668 & -75.3704 & 5409.499 \\
2GVG & 2344 & -196070 & -83.6476 & 6144.705 \\
2GVL & 2344 & -115955 & -49.4689 & 7256.643 \\
2H3B & 2344 & -156773 & -66.8826 & 5304.75 \\
3DGR & 2344 & -184425 & -78.6798 & 4800.213 \\
3DHD & 2344 & -186579 & -79.5986 & 6195.604 \\
3DKJ & 2344 & -191112 & -81.5322 & 6340.178 \\
3DKL & 2344 & -152832 & -65.2012 & 5911.7 \\
\hline
\end{tabular}

ANOVA of 1172 drug candidates (IGEMDock Data).

\begin{tabular}{lcccccc}
\multicolumn{8}{c}{ ANOVA } \\
\hline Source of Variation & SS & $d f$ & MS & $F$ & P-value & F crit \\
\hline Between Groups & 2328693 & 10 & 232869.3 & 39.43557 & $6.27 \mathrm{E}-78$ & 1.83107 \\
Within Groups & $1.52 \mathrm{E}+08$ & 25773 & 5905.057 & & & \\
Total & $1.55 \mathrm{E}+08$ & 25783 & & & & \\
\hline
\end{tabular}

Breakdown of drug candidates by types (IGEMDock Data).

\begin{tabular}{ccc} 
Classification & Average & St. Dev \\
\hline Alkaloids & -88.31890148 & 12.78578401 \\
Flavonoids & -92.04583914 & 15.64317212 \\
Imides & -89.20714988 & 10.78330885 \\
Lactams & -86.50791125 & 13.02275967 \\
Lactones & -80.13076378 & 67.6932637 \\
NSAIDs & -91.34529884 & 10.11470257 \\
Sulf & -84.68074545 & 8.485802076
\end{tabular}


Drug candidates by types (IGEMDock Data).

\begin{tabular}{|c|c|c|}
\hline \# of Drugs & Drug Title & Energy \\
\hline \multirow[t]{2}{*}{1} & FDA - 99-1 & -136.84 \\
\hline & FDA - 99-0 & -135.57 \\
\hline \multirow[t]{2}{*}{2} & FDA - 570-0 & -146.57 \\
\hline & FDA - 570-1 & -145.65 \\
\hline \multirow[t]{2}{*}{3} & FDA - 266-0 & -135.91 \\
\hline & FDA - 266-1 & -135.77 \\
\hline \multirow[t]{2}{*}{4} & FDA - 446-1 & -153.41 \\
\hline & FDA - 446-0 & -151.65 \\
\hline \multirow[t]{2}{*}{5} & FDA - 182-1 & -152.83 \\
\hline & FDA - 182-0 & -149.23 \\
\hline \multirow[t]{2}{*}{6} & FDA - 533-1 & -130.52 \\
\hline & FDA - 533-0 & -129.69 \\
\hline \multirow[t]{2}{*}{7} & FDA - 288-0 & -128.29 \\
\hline & FDA - 288-1 & -128.02 \\
\hline \multirow[t]{2}{*}{8} & Vincristine-1-1 & -122.65 \\
\hline & Vincristine-1-0 & -116.12 \\
\hline \multirow[t]{2}{*}{9} & Etoposide-1-0 & -122.01 \\
\hline & Etoposide-1-1 & -120.41 \\
\hline \multirow[t]{2}{*}{10} & Alkaloids - $504-0-1$ & -117.91 \\
\hline & Alkaloids - $504-0-0$ & -114.93 \\
\hline
\end{tabular}

Summary of Control Drugs (IGEMDock Data).

\begin{tabular}{c|c}
\multicolumn{2}{c}{ Visfatin Control } \\
\hline Low Value & -37.9203 \\
High Value & 470.4448 \\
\hline Average & 9.625877 \\
Standard Deviation & 104.82
\end{tabular}


Dragon Data of Visfatin Control Molecules.

\begin{tabular}{c|c|c|c} 
NAME & MW & MLOGP & MLOGP2 \\
\hline 3G8E_IS1_A_501 & 486.3 & 2.456 & 6.031 \\
3DKL_UNU_A_504 & 114.1 & 1.308 & 1.711 \\
3DKL_PRP_B_503 & 377 & -0.664 & 0.441 \\
3DKJ_UNU_A_502 & 114.1 & 1.308 & 1.711 \\
3DKJ_PRP_A_501 & 377 & -0.664 & 0.441 \\
3DHD_NMN_A_503 & 319.1 & -0.067 & 0.004 \\
3DGR_A12_A_501 & 408.1 & -0.57 & 0.325 \\
2H3D_NMN_A_3819 & 319.1 & -0.067 & 0.004 \\
2H3B_SO4_A_1000 & 96.07 & -2.399 & 5.755 \\
2GVJ_DGB_A_502 & 362.3 & 3.333 & 11.108 \\
2GVG_NMN_A_501 & 319.1 & -0.067 & 0.004 \\
2GVG_NMN_C_503 & 319.1 & -0.067 & 0.004 \\
2GVG_NMN_E_505 & 319.1 & -0.067 & 0.004 \\
2G97_DGB_A_1001 & 362.3 & 3.333 & 11.108 \\
2G96_NMN_A_1001 & 319.1 & -0.067 & 0.004 \\
2E5D_NCA_A_1501 & 116.1 & 0.119 & 0.014 \\
2E5C_PRP_A_902 & 377 & -0.664 & 0.441
\end{tabular}

Dragon Data of Visfatin drug candidates.

\begin{tabular}{c|c|c|c} 
NAME & MW & MLOGP & MLOGP2 \\
\hline FDA 2 - 446 & 359.04 & 0.364 & 0.133 \\
FDA 2 - 570 & 312.211 & -0.009 & 0 \\
FDA 2 - 266 & 254.15 & 0.372 & 0.139 \\
FDA 2 - 182 & 516.61 & 0.391 & 0.153 \\
FDA 2 - 533 & 265.13 & 3.174 & 10.076 \\
FDA 2 - 99 & 504.72 & 2.029 & 4.117
\end{tabular}

\section{Discussion}

Multiple compounds were identified as effective based upon their interactions with each protein. Specifically an average energy of -133.699 was found for the drug candidates compared to 9.625877 for the control molecules. There were 4 control molecule which seemed to be an outliers with values of $39.8249,87.6701,99.1493$, and 470.4448 . Without these outliers the average decreases to -23.3034. An ANOVA to determine differences between the 
11 proteins analyzed indicated major differences with a $F$ value of 39.43557 compared to $F$ critical value of 1.831. A search of the data indicates that 3DKL shows the greatest differences. Grouping analysis will be used to understand differences in protein active sites. 10 drugs were chosen due to their low binding energies (for both binding interactions). Structural analysis found that many of these molecules are small with similar partition coefficient (-0.009 to 3.174) of the top binders similar to that of the control molecules.

\section{Conclusion}

By using the computational techniques we were able to identify several molecule that show improved binding efficacy over currently used Visfatin inhibitors. These Visfatin drug candidates indicated a diverse pool of Visfatin binders with improved efficacy. This work can be used to engineer these motifs into novel Visfatin inhibitors for improved drug efficacy.

\section{Acknowledgments}

This Project was supported by The College of Natural Science and Mathematics at The Richard Stockton College of New Jersey.

\section{References}

1. Cinar, N.; Gurlek, A., Association between novel adipocytokines adiponectin, vaspin, visfatin, and thyroid: An experimental and clinical update. Endocr Connect 2, (4), R30-8.

2. Shrestha, C.; He, H.; Liu, Y.; Zhu, S.; Xiong, J.; Mo, Z., Changes in Adipokines following Laparoscopic Roux-en-Y Gastric Bypass Surgery in Chinese Individuals with Type 2 Diabetes Mellitus and BMI of 22-30 kg.m(-2.). Int J Endocrinol 2013, 240971.

3. Al-Suhaimi, E. A.; Shehzad, A., Leptin, resistin and visfatin: the missing link between endocrine metabolic disorders and immunity. Eur J Med Res 18, 12.

4. Lu, H.; Gauvreau, D.; Tom, F. Q.; Lapointe, M.; Luo, X. P.; Cianflone, K., Inflammatory markers and adipokines alter adipocyte-derived ASP production through direct and indirect immune interaction. Exp Clin Endocrinol Diabetes 121, (4), 194-200.

5. Mazaherioun, M.; Hosseinzadeh-Attar, M. J.; Janani, L.; Vasheghani Farahani, A.; Rezvan, N.; Karbaschian, Z.; Hossein-Nezhad, A., Elevated serum visfatin levels in patients with acute myocardial infarction. Arch Iran Med 15, (11), 688-92.

6. Mannino, G. C.; Sesti, G., Individualized therapy for type 2 diabetes: clinical implications of pharmacogenetic data. Mol Diagn Ther 16, (5), 285-302.

7. Skvarca, A.; Tomazic, M.; Krhin, B.; Blagus, R.; Janez, A., Adipocytokines and insulin resistance across various degrees of glucose tolerance in pregnancy. $J$ Int Med Res 40, (2), 5839.

8. Kang, Y. S.; Cha, D. R., The role of visfatin in diabetic nephropathy. Chonnam Med J 47, (3), 139-43.

9. Derosa, G.; Cicero, A. F.; D'Angelo, A.; Fogari, E.; Maffioli, P., Effects of 1-year orlistat treatment compared to placebo on insulin resistance parameters in patients with type 2 
diabetes. J Clin Pharm Ther 37, (2), 187-95.

10. Sonoli, S. S.; Shivprasad, S.; Prasad, C. V.; Patil, A. B.; Desai, P. B.; Somannavar, M. S., Visfatin--a review. Eur Rev Med Pharmacol Sci 15, (1), 9-14. 